and I should certainly agree with the observers quoted by Mr. Kellett Smith that large doses of this drag may produce, or at least powerfally aid in producing, "black water," as I have pointed out that about one-fifth of the ordinary acid sulphate of quinine is zanthin, which is physiologically and pathologically equivalent to uric acid, so that large doses may add very greatly to the quantity of this substance available for solution in the blood and thus bring about the destruction of red cells observed in men and animals. I may also perhaps be allowed to remind Mr. Kellett Smith and others who may be interested in these matters that my own blood (and I believe that of everyone) varies its quality from day to day, even from hour to hour, with the amount of uric acid passing through it into the urine.

I have written of " black water" in connexion with the points brought out by my former colleague Dr. Wheaton in a paper in the Transactions of the Pathological Society, vol. xlvi., and I shall not ask for space to consider other points on which I have written at length elsewhere. Bat with reference to Mr. Kellett Smith's closing paragraph I should like to make the practical suggestion that living on a diet which is free from uric acid and using salicylates between attacks to sweep out the uric acid accumulated in the spleen and elsewhere, as well as that introduced in the form of quinine, might very greatly reduce the amount of available uric acid and mitigate or prevent such troublesome symptoms and sequelæ as congestion of the liver and spleen, vomiting, diarrhoea, and other "bilious" troubles, and even the "black water" itself. And some of these points I have been able to demonstrate practically in the persons of members of the profession who have returned from various malarial countries and have taken the trouble to follow $\mathrm{my}$ suggestions.

I am, Sirs, yours faithfully,

Brook-street, W., March 28th, 1898. ALEXANDER HAIG.

CREASOTAL AND GUAIACOL CARBONATE: TWO SPECIFIC REMEDIES AGAINST TUBERCULOSIS.

To the Editors of THE LANOET.

SIRs,-In The LANCET of Jan. 22nd, 1898, Dr. Chaumier has published an article on "Creasote and Some of its Derivatives." Dr. Chaumier recommends that creasotal be taken by teaspoonfuls and considers the use of the drug contra-indicated only in fever and diarrhoea. According to the experiences, however, which we have been made acquainted with by the publications of the University Clinics of Berlin, Vienna, and Munich these contra-indications do not exist; on the contrary, the action of creasotal and guaiacol carbonate is very favourable upon the fever of phthisical patients, this disappearing after a relatively short period of treatment with these drugs. The cause by which Dr. Chaumier was led to believe these drugs contra-indicated in fever probably lies in the fact that he employed larger doses than were necessary. It is neither necessary nor is it useful to take the creasotal by teaspoonfuls. The same remedial effects are obtained with the administration by drops, as introduced by Professor von Leyden of Berlin. These small doses, besides being less expensive, are also much better borne by all patients, whereas the strong doses as used by Dr. Chaumier cannot be borne by many patients.

The Charitê Annalen (Berlin, 1897) recently contained an interesting report on the results obtained with creasotal (creasote carbonate) in the Universily Clinic of Professor von Leyden. The conclusions arrived at by the experiments made under the direction of the chief surgeons of this clinic show that the remedial effect of the drug is not a merely symptomatic, but a specific one. After the administration of creasote, which drug was formerly also used by Professor von Leyden, it has always been noticed that the appetite disappeared, the general health grew worse, and symptoms of intense disturbance of the stomach and intestines soon followed. The creasotal was free from these noxlous by-effects. The following mode of administration was adopted. Each patient began with five drops three times daily, increasing the dose three drops every day until twenty. five drops were taken at a dose. At this they were kept for from one to four weeksin some cases even for several months; then the dose was diminished in a similar ratio until only ten drops were taken thrice daily, and then eventually the ascending scale was egun again.

The results obtained in von Leyden's clinic are fully detailed in the above-mentioned Charité Annalen. Though the greater part of the treatment was carried out during the winter months, when the climatic influences were unfavourable, very good results were nevertheless obtained. The general condition of the patients was markedly improved; fever, night sweats, and all the bad symptoms disappeared entirely after six weeks of treatment; the appetite rapidly increased. Even in cases where the patients had taken creasote before with the effect of causing a complete loss of appetite, under creasotal the appetite increased from week to week. Very much the same thing can be said of the weight, in which up to $16 \mathrm{lb}$. were gained in three months. Upon the night sweats and the fever the action of creasotal was very favourable. Cough and expectoration gradually diminished and finally disappeared altogether Wherever the treatment was continued for over six months marked improvement of the local condition was noticed The lungs in these cases were partly cured. In some cases the physical symptoms of phthisis disappeared entirely after a treatment of from six to eight months, so that a perfect care was obtained and the lungs of these patients were perfectly healed and quite normal again. Considering that in these cases only 300 grammes of creasotal were required to obtain a perfect cure and that physicians can obtain from their druggists $1 \mathrm{oz}$. of creasotal at the average price of 2s., it will appear that the expenditure for the remedy, distributed over several months, is no drawback, and that also the poorest patients can afford to take part in the benefits of the drug.

In the report of Professor von Leyden's clinic the anthor concludes by saying: We think we are entitled to assume a specific action of creasotal. Reviewing our experiences we come to the opinion that any case of incipient or not too far advanced phthisis may be treated with creasotal with the expectation of a good result. Naturally a nourishing diet and general good hygiene must go hand in hand with it. And here is the chief advantage of creasotal over creasote, inasmuch as it improves the appetite and does not irritate the gastro-intestinal canal it permits us to enforce the proper dietetic treatment of the disease at the same time Very much the same favourable views in regard to creasotal are expressed in the recently published reports of the university clinics of Vienna and Munich. ${ }^{2}$ I believe these experiences prove that Dr. Chaumier's objection to the administration of creasotal in fever is deprived of foundation. As above mentioned the presence of fever is, perhaps, to be considered as a contra-indication for the enormous doses of creasotal which Dr. Chaumier employs, while on the other hand the small doses as recommended by Professor von Leyden effect the disappearance of the fever.

In The Lancers of Aug. 8th, 1896, Dr. F. R. Walters expressed his opinion to the effect that creasotal is an excel. lent remedy against tuberculosis, but that it appears to be too expensive as compared with creasote. I believe also this opinion cannot be agreed with. Here in Germany at least the patient buys the creasote in form of creasotal considerably cheaper than creasote pills and capsules. It must be borne in mind that the creasotal is administered by drops in liquid form, whereas the creasote can rarely be taken in a pure state, but is dispensed. in the form of mixtures or capsules. By this its cost is increased so much that creasote in the cheapest emulsion-viz, tinct. creasoti-costs more than twice as much as an equivalent quantity of creasote in the form of creasotal. In the form of capsules creasote is still more expensive. I believe in England there will be an analogous proportion. In consideration of this fact it appears also that from an economical point of view the creasotal treatment is to be preferred to the administration of creasote in tinctures or capsules, so that in every respect the balance is greatly in favour of the new method. I am, Sirs, yours faithfully,

Radebeul, Germany. R. SEIFERT,

\section{AN UNKNOWN LARVAL PARASITE.}

To the Editors of THE LANOET.

SIRS,-There has recently come under my notic $\theta$ a parasitic affection of the skin of which I can find no mestion in text books, and I should be glad if any of your readers who bare

1 Vide Professor Ziemssen's Annalen der Mụnchner Krankenhàuset. 